\title{
Grußwort zum 80. Geburtstag von em. o. Univ. Prof. Dipl.-Ing. Dr. mont. Herbert Hiebler
}

\author{
Johannes Schenk \\ Leoben, Österreich
}

Online publiziert am 10. März 2015

Die Berg- und Hüttenmännischen Monatshefte und ASMET gratulieren im Namen aller Leser Prof. Herbert Hiebler sehr herzlich zu seinem 80 . Geburtstag. Er ist seit seinem Studium mit der Montanuniversität Leoben und dem Lehrstuhl für Eisen- und Stahlmetallurgie verbunden und hat deren Entwicklung durch seinen persönlichen Einsatz und Engagement mit geprägt. Jahrelang war er auch verantwortlich für die Herausgabe der Berg- und Hüttenmännischen Monatshefte.

Für dieses Heft haben ehemalige Mitarbeiter und Kollegen mehrere Originalbeiträge verfasst, als Reminiszenz zu seinen beruflichen Leistungen und persönlichen Engagement auf dem Gebiet der Eisen- und Stahlmetallurgie. In seiner aktiven Zeit als Professor des Instituts für Eisenhüttenkunde (heute Lehrstuhl für Eisen- und Stahlmetallurgie) zwischen 1979 und 2001 waren seine Arbeitsschwerpunkte im Bereich der Schmelzreduktion von Eisenerzen mit Wasserstoff, Schlackenmetallurgie bei der Roheisen- und Stahlerzeugung, Stranggießmetallurgie und Wärmebehandlung. Dies zeigt das universelle Interesse und Wissen von Prof. Hiebler im Fachbereich der Eisen- und Stahlmetallurgie. Seit seiner Emeritierung hat er sich sehr um die Montangeschichte in der Steiermark verdient gemacht. Die Restaurierung des Radwerks IV in Vordernberg wurde unter seiner Führung initiiert und erfolgreich abgeschlossen.

Unter seinen zahlreichen anerkannten Publikationen sind besonders seine Beiträge als Herausgeber von zwei
Bänden des metallurgischen Standardwerkes "GmelinDurrer: Metallurgy of Iron" hervorzuheben. Auch die Lehre und Förderung der Studenten war ihm stets ein großes Anliegen. Einer ganzen Generation von Hüttenleuten gab er das erforderliche fachlicheWissen für ihre Aufgaben und Karriereentwicklung in Wirtschaft und Wissenschaft mit. Verdiente Führungskräfte in der Industrie und anerkannte Forscher waren einst seine Schüler.

Seine fachliche und wissenschaftliche Kompetenz war bei der Stahlindustrie stets anerkannt und gefragt. Er war deshalb auch von 1988 bis 2000 Mitglied des Aufsichtsrates der voestalpine Stahl Donawitz $\mathrm{GmbH}$ und dabei ab 1991 stellvertretender Vorsitzender.

Große Verdienste hat sich Prof. Hiebler für die Entwicklung des Vereines der Eisenhütte Österreich - heute ASMET - gemacht, den er viele Jahre als Vorstand führte. Auf seine Initiative hin wurde vor 20 Jahren die Studentensektion der ASMET gegründet.

Aus den genannten Gründen möchten wir Prof Hiebler unseren Dank aussprechen und ihm diese Ausgabe zu seinem 80. Geburtstag widmen.

Wir wünschen Ihnen, lieber Herr Professor Herbert Hiebler, noch viele schöne Jahre und vor allem Gesundheit.

Glückauf!

Johannes Schenk

Bruno Hribernik 